\title{
Subamniotic Hemorrhage, a Possible New Presentation of Fetal and Neonatal Alloimmune Thrombocytopenia
}

\author{
Steven Giesbers ${ }^{\mathrm{a}}$ Manon Bos ${ }^{\mathrm{b}} \quad$ Johan Bulten $^{\mathrm{c}} \quad$ Lotte van der Meeren $^{\mathrm{b}, \mathrm{d}} \quad$ Joris van Drongelen ${ }^{\mathrm{a}}$ \\ aDepartment of Gynaecology and Obstetrics, Radboud University Medical Centre, Nijmegen, The Netherlands; \\ ${ }^{b}$ Department of Gynaecology and Obstetrics, Department of Pathology, Leiden University Medical Centre, Leiden, The \\ Netherlands; ' Department of Pathology, Radboud University Medical Centre, Nijmegen, The Netherlands; ${ }^{\mathrm{d} D e p a r t m e n t}$ \\ of Pathology, University Medical Centre Utrecht, Utrecht, The Netherlands
}

\section{Established Facts}

- Fetal and neonatal alloimmune thrombocytopenia (FNAIT) is a rare fetal disease in which maternal antibodies directed toward fetal human platelet antigens (HPA) are formed during pregnancy and cause fetal thrombocytopenia.

- FNAIT is a condition which generally manifests postnatally with petechiae or hematomas, alongside the presence of allo-antibodies directed to fetal HPA, in maternal serum.

- Rarely the diagnosis of FNAIT is made prenatally by ultrasound detection of a fetal intracerebral hemorrhage or with hemorrhage in other fetal organs such as the gastrointestinal tract.

\section{Novel Insights}

- This is the first publication suggesting that a subamniotic hemorrhage was due to fetal and neonatal alloimmune thrombocytopenia (FNAIT).

- More data are needed before FNAIT should be considered as a diagnosis when fetal ultrasound reveals a placental hematoma on the fetal side.

\section{Keywords}

Human platelet antigens-5b · Allo-antibodies · Placenta . Subamniotic $\cdot$ Hematoma $\cdot$ Fetal and neonatal alloimmune thrombocytopenia

\section{Abstract \\ Background: Fetal and neonatal alloimmune thrombocyto- penia (FNAIT) is a rare fetal disease in which maternal anti- bodies directed toward fetal human platelet antigens (HPA)}

are formed during pregnancy and cause fetal thrombocytopenia. The diagnosis FNAIT is suspected when a fetus or neonate presents with signs of bleeding. Case: We describe a pregnancy complicated by a placental hematoma in the 20th week of gestation as the first manifestation of FNAIT. Further evaluation showed signs of germinal matrix hemorrhage and HPA-5b allo-antibodies. After the diagnosis, intravenous immunoglobulin was administered weekly and a healthy daughter was born at 37 weeks. Histopathological analysis revealed that the hematoma was caused by a sub-
(C) 2022 The Author(s).

Published by S. Karger AG, Basel

This is an Open Access article licensed under the Creative Commons Attribution-NonCommercial-4.0 International License (CC BY-NC) (http://www.karger.com/Services/OpenAccessLicense), applicable to the online version of the article only. Usage and distribution for commercial purposes requires written permission.
Correspondence to:

Steven Giesbers, stevengiesbers@gmail.com

Joris van Drongelen, joris.vandrongelen@ radboudumc.nl 
amniotic hemorrhage of fetal origin. Conclusion: A subamniotic hematoma appears to be the first manifestation of FNAIT.

(c) 2022 The Author(s).

Published by S. Karger AG, Basel

\section{Introduction}

Fetal and neonatal alloimmune thrombocytopenia (FNAIT) is a rare disease in which maternal antibodies are formed to fetal human platelet antigens (HPA) [1]. FNAIT is the predominant cause of thrombocytopenia in an otherwise healthy fetus [2]. The clinical diagnosis is based on thrombocytopenia or signs of bleeding in the fetus or neonate, alongside the presence of allo-antibodies directed to fetal HPA, in maternal serum [2]. The incidence of FNAIT is between 1 in 1,000 and 10,000 births [3]. Bleeding symptoms vary from minor skin bleeding to severe organ bleeding. Intracranial hemorrhage (ICH) is the most concerning complication of FNAIT as it may lead to perinatal death or lifelong neurologic sequelae [4, 5]. The incidence of ICH is found to be at least 10 per 100,000 newborns [3]. When FNAIT is suspected during pregnancy due to signs of fetal hemorrhage, treating with weekly maternal IV immunoglobulin administration results in a better outcome [2]. The most common antigen found in FNAIT in Caucasians is HPA-1a $( \pm 80 \%)$, HPA$5 \mathrm{~b}( \pm 15 \%)$, and HPA $-3 \mathrm{a}( \pm 2 \%)[1]$.

\section{Case Presentation}

A 30-year-old multiparous woman (G3P2) with no medical, obstetric, and family history was referred to our tertiary center. She had no history of previous blood transfusion. Her two previous pregnancies, with the same partner, were uneventful, with two spontaneous vaginal deliveries. At 19+4 weeks gestation, she had undergone routine mid-trimester ultrasound screening for structural anomalies, at which a placental lesion measuring $71 \times 45 \times 59 \mathrm{~mm}$ was seen.

The advanced ultrasound at the Prenatal Diagnostics and Therapy department (Radboud University Medical Centre, Nijmegen, The Netherlands) at 20+4 weeks gestation showed an echolucent lesion on the surface of the placenta (shown in Fig. 1) with the umbilical cord insertion positioned just at the height of this lesion (shown in Fig. 2). The differential diagnosis for an echolucent placental lesion includes a subamniotic hematoma, a subchorionic hematoma, placental and umbilical cord (pseudo-)cyst, a (partial) molar pregnancy, a chorangioma, or a chorangiocarcinoma. Within the lesion, no flow was seen with color doppler, which excluded the possibility of a chorangioma. Fetal biometry was consistent with gestational age, and no fetal anomalies were detected. Doppler evaluation showed a peak systolic velocity (PSV) in the middle cerebral artery (MCA) at 1.4 MoM, just below the threshold for characterizing fetal anemia $(<1.5 \mathrm{MoM})[6]$.

Subamniotic Hemorrhage in FNAIT

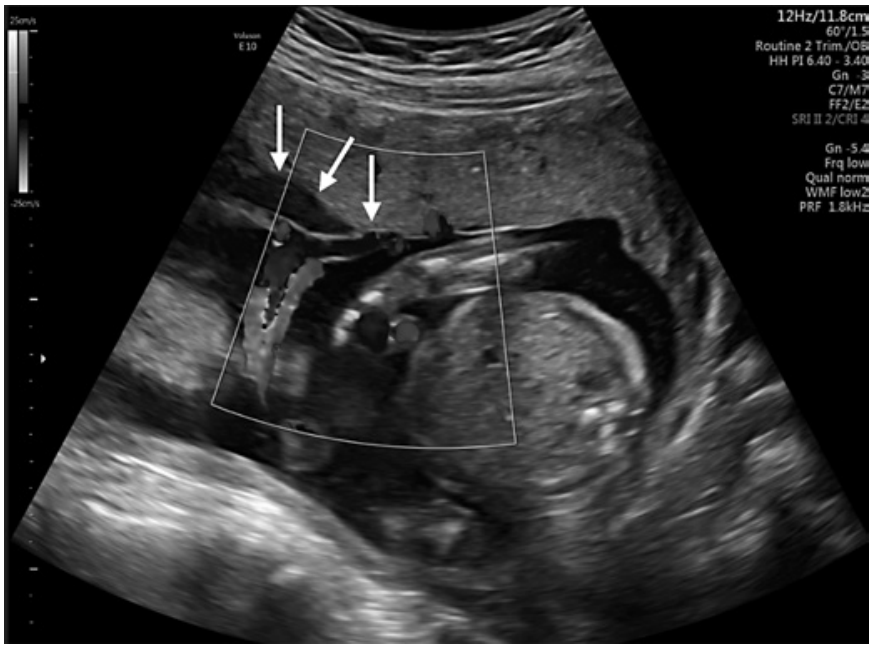

Fig. 1. Ultrasound image at $19+4$ weeks of pregnancy showing the subamniotic lesion (arrows) located next to the umbilical cord insertion of the placenta. Within the subamniotic lesion, no flows were detected using doppler.

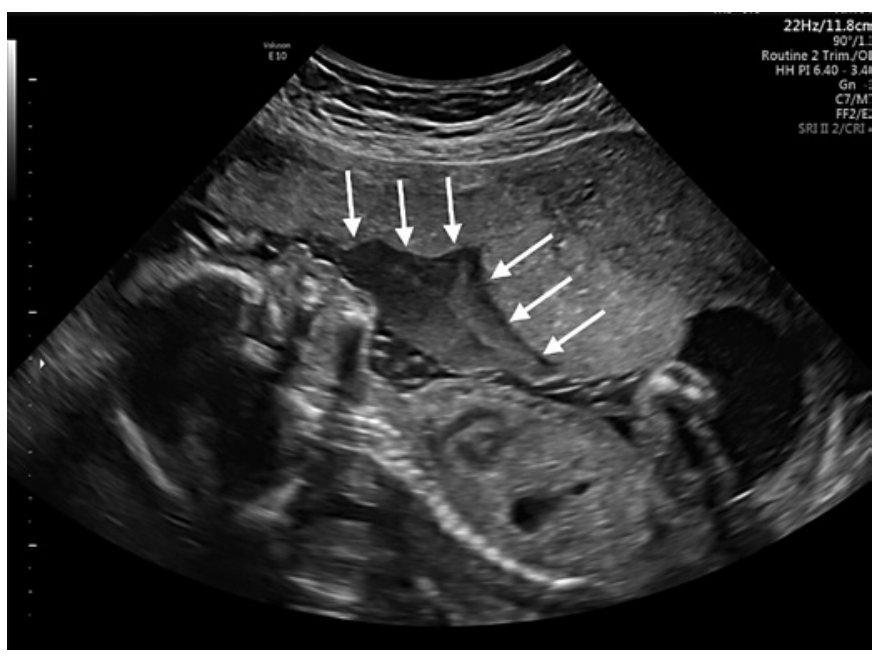

Fig. 2. Ultrasound image at $19+4$ weeks of pregnancy showing the subamniotic lesion (arrows).

Because of the lack of flow inside the lesion, the position of the hematoma, and the possibility of fetal anemia (PSV in MCA at 1.4 MoM), a subamniotic hematoma was considered most likely the cause. There was no indication that a trauma could be the cause of the bleeding and FNAIT was suggested as an underlying mechanism. Laboratory evaluation showed maternal HPA-5b allo-antibodies and amniocentesis confirmed that the fetus was HPA-5bantigen positive. A week later, PSV of the MCA was reduced (1.0 $\mathrm{MoM})$. In the meantime, a fetal magnetic resonance imaging (MRI) scan of the brain was performed to look for signs of ischemic cerebral damage due to a possible hypovolemic shock by the 
Fig. 3. Fetal MRI image at $22+1$ (a) and $27+5$ (b) weeks of pregnancy suggestive for germinal matrix hemorrhage (arrow) on both sides.
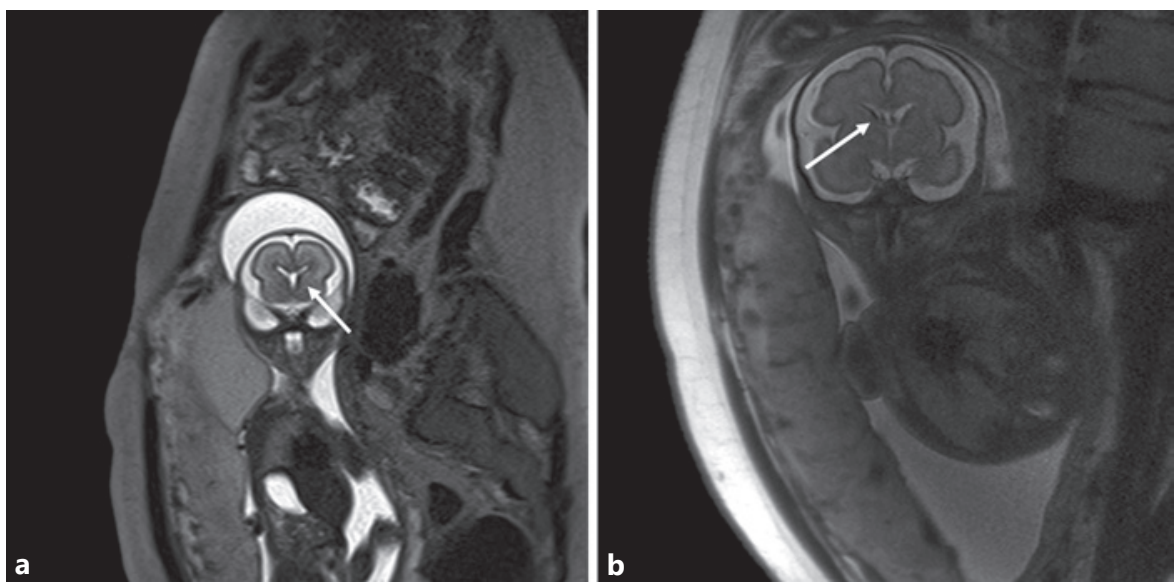

fetal bleeding. The MRI did not reveal any signs of hypoxic cerebral tissue loss; however, it did suggest that a germinal matrix hemorrhage may have occurred previously (shown in Fig. 3). Due to the strong suspicion of a subamniotic bleeding caused by FNAIT, weekly administration of intravenous immunoglobulin $(1 \mathrm{mg} / \mathrm{kg})$ was started at $22+2$ weeks of gestation. The fetal MRI was repeated twice at $25+3$ weeks and $27+5$ weeks gestation demonstrating a normal fetal brain development. Ultrasound examination throughout pregnancy showed normal fetal interval growth and normal dopplers.

Because of the risk of fetal bleeding due to FNAIT, labor was induced at 37 weeks of pregnancy. Given the history of two uncomplicated deliveries, vaginal delivery was considered to be the appropriate mode of delivery. Due to the diagnosis of FNAIT, fetal scalp blood sampling, a scalp electrode or vaginal operative delivery were contraindicated. She had an uncomplicated delivery and gave birth to a healthy daughter. The third stage of labor and the postpartum period were uneventful. The newborn had Apgar scores of 9 and 10 after, respectively, 1 and $5 \mathrm{~min}$ and weighted $2,910 \mathrm{~g}$ (p20-p50). Venous umbilical cord blood analysis showed a $\mathrm{pH}$ of 7.29 , a lactate of $2.9 \mathrm{mmol} / \mathrm{L}$. The platelet count immediately after birth, measured from the umbilical cord blood, was 274 $\times 10^{-9} / \mathrm{L}$ (normal range $210-650 \times 10^{-9} / \mathrm{L}$ ). This was initially monitored daily by the neonatologist dropping to $183 \times 10^{-9} / \mathrm{L}$ on day 3 after birth. There were no signs of neurologic damage due to intracranial bleeding, and there were no hematoma or petechia visible. The neonate was discharged from the hospital 5 days after birth. Platelet counts were then monitored weekly for 1 month by the pediatrician and recovered to the normal range.

Macroscopic examination of the placenta showed a large subamniotic hematoma comprising approximately $25-30 \%$ of the placental volume as was seen on ultrasound at $19+4$ weeks of gestation (shown in Fig. 4a). Histologically this hematoma consisted mainly of degenerated blood with fibrin and nucleated fetal erythrocytes under the amnion. The placental parenchyma had a normal maturation with focal ischemia, a low-grade chronic villitis of unknown etiology. Apart from the subamniotic hematoma, a subchorionic hematoma was present in the maternal compartment. The nucleated fetal erythrocytes were also visualized by an immunohistochemical staining for fetal gamma-globulin (CD71, shown in Fig. 4c).

\section{Discussion}

A subamniotic hematoma is a rare finding and, in this case, could have been the first presentation of FNAIT. To the knowledge of the authors, this is the first publication of a subamniotic hemorrhage due to FNAIT. A subamniotic hematoma is defined as a hemorrhage between the amniotic membrane and the fetal chorionic plate, following damage to one of the branches of an umbilical vessel. Most cases are reported at the time of delivery and are thought to be caused by traction on the umbilical cord [7]. However, subamniotic hemorrhage is also known to occur antenatally [8]. Subamniotic hematomas that occur during pregnancy are associated with adverse fetal outcome such as fetal growth restriction, fetal distress, and perinatal death due to fetal anemia $[8,9]$. The fetal outcome is mainly based on the size of the bleeding. Due to the size of the hematoma in our case, the fetus would not have survived if it lost the volume over a short time. Based on this, we think that the bleeding occurred over a long period, giving the fetus time to hemodynamically deal with and recover from the blood loss. Due to the absence of other possible causes for a subamniotic hematoma and the presence of HPA-5b allo-antibodies, we think that FNAIT is most likely the cause of the subamniotic hemorrhage.

FNAIT is a condition which generally manifests postnatally with petechiae or hematomas [2]. Rarely the diagnosis of FNAIT is made prenatally by ultrasound detection of a fetal intracerebral hemorrhage or with hemorrhage in other fetal organs such as the gastrointestinal tract [10]. Even more rare was the presentation in our case, but the combination of the hematoma, the PSV in the MCA was at the upper limit of normal and the HPA$5 \mathrm{~b}$ allo-antibodies convinced us of the diagnosis. Postpar- 

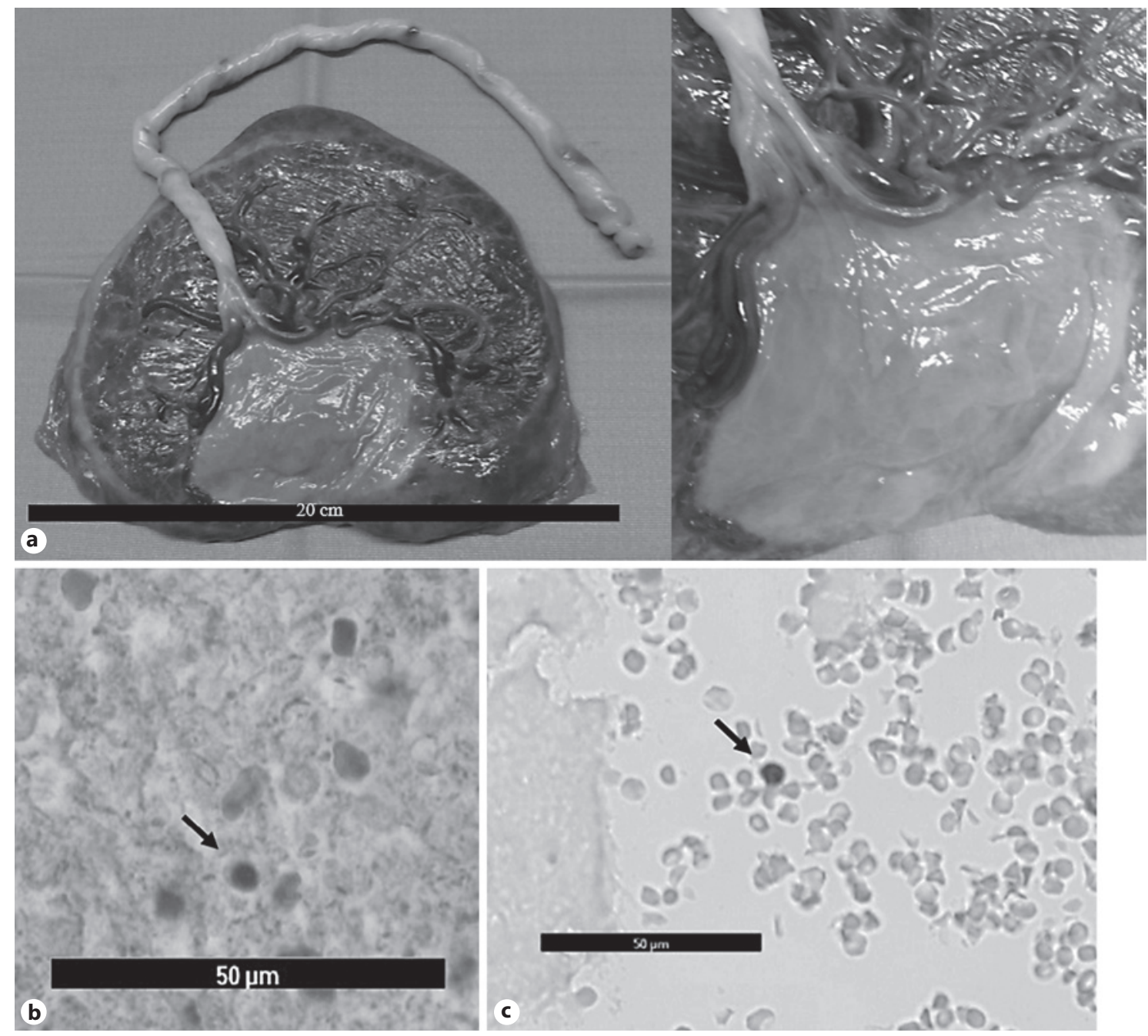

Fig. 4. Examination of the placenta. a Macroscopic image of the fetal side of the placenta with a subamniotic hematoma. The placenta weighted $382 \mathrm{~g}(<\mathrm{p} 10)$, and the subchorionic hematoma consisted of $25-30 \%$ of the placenta volume. $\mathbf{b}$ Histologic image of nucleated red blood cells (arrow) found in the subchorionic hematoma. c Histologic image after immunohistochemical staining for fetal gamma-hemoglobin (CD71) identifying fetal red blood cells (arrow) in the subchorionic hematoma.

tum diagnostic tests: both the follow-up of the blood results of the neonate and the histology of the placenta, confirmed the diagnosis FNAIT and implies that clinicians may give FNAIT due consideration as part of a differential work up once a placental hematoma is diagnosed prenatally. Diagnosing FNAIT prenatally has the potential to prevent additional adverse events.

The fetus also showed bilateral germinal matrix bleeding, which could have led to an adverse neonatal outcome. It is likely that this is related to fetal thrombocytopenia induced by FNAIT. It is therefore very important that further cases of this kind of placental hematomas recognized on ultrasound examination are reported before FNAIT is considered as the cause. There is a risk of major bleeding in other organs, especially the brain. Finally, FNAIT can occur in subsequent pregnancies, often with worse consequences. The effects of the FNAIT on subsequent pregnancies can be reduced by treating the mother with weekly IVIG administration which significantly reduces the chance of ICH [2]. The delivery must take place in a NICU center. Depending on obstetric considerations, vaginal delivery may be appropriate, but operative vaginal delivery is contraindicated due to the risk of ICH. 
With this article, we hope to create awareness to consider FNAIT as a possible diagnosis when fetal ultrasound reveals a placental hematoma on the fetal side. We believe that the early recognition and treatment of FNAIT in our case contributed to the successful outcome.

\section{Acknowledgment}

The authors thank Leo Gurney for reviewing the article as a native speaker.

\section{Statement of Ethics}

We have obtained permission from all individuals named in the acknowledgments. Written consent has been obtained from the patient for publication of the details of their medical case and any accompanying images. This is documented in the electronic patient record, which is also accessible to the patient.

\section{Conflict of Interest Statement}

The authors have no conflicts of interest to declare.

\section{Funding Sources}

No financial support or funding was received for this article.

\section{Author Contributions}

Steven Giesbers has written the draft of this manuscript and took care of the submission to the journal. Manon Bos took care of the placental histology and staining and has written the part of the article about this, including the figures. Hans Bulten was involved from the very beginning. He took care of the placenta and arranged the right slides. He was also involved in the thinking process about the origin of the bleeding. Lotte van der Meeren supervised Manon Bos and was involved in the staining of the placenta. Joris van Drongelen supervised Steven Giesbers in writing the article and was involved from the very beginning and in all the thinking about this case. He was also the direct contact with the patient.

\section{Data Availability Statement}

The data that support the findings of this study are not publicly available due to the fact that this information could compromise the privacy of research participants but are available from the corresponding author [J.D.].

\section{References}

1 Risson DC, Davies MW, Williams BA. Review of neonatal alloimmune thrombocytopenia. J Paediatr Child Health. 2012;48(9):816-22.

2 de Vos TW, Winkelhorst D, de Haas M, Lopriore E, Oepkes D. Epidemiology and management of fetal and neonatal alloimmune thrombocytopenia. Transfus Apher Sci. 2020;59(1):102704

3 Kamphuis MM, Paridaans NP, Porcelijn L, Lopriore E, Oepkes D. Incidence and consequences of neonatal alloimmune thrombocytopenia: a systematic review. Pediatrics. 2014; 133(4):715-21.
4 Winkelhorst D, Kamphuis MM, de Kloet LC, Zwaginga JJ, Oepkes D, Lopriore E. Severe bleeding complications other than intracranial hemorrhage in neonatal alloimmune thrombocytopenia: a case series and review of the literature. Transfusion. 2016;56(5):1230.

5 Winkelhorst D, Kamphuis MM, Steggerda SJ, Rijken M, Oepkes D, Lopriore E, et al. Perinatal outcome and long-term neurodevelopment after intracranial haemorrhage due to fetal and neonatal alloimmune thrombocytopenia. Fetal Diagn Ther. 2019;45(3):184-91.

6 Pretlove SJ, Fox CE, Khan KS, Kilby MD. Noninvasive methods of detecting fetal anaemia: a systematic review and meta-analysis. BJOG. 2009;116(12):1558-67.
7 Van Den Bosch T, Van Schoubroeck D, Cornelis A, Dubin M. Prenatal diagnosis of a subamniotic hematoma. Fetal Diagn Ther. 2000; 15(1):32-5

8 Owada M, Shibata Y, Suzuki S. Case series of intrauterine subamniotic hemorrhage. Case Rep Obstet Gynecol. 2019;2019:1828457.

9 Windrim C, Athaide G, Gerster T, Kingdom JCP. Sonographic findings and clinical outcomes in women with massive subchorionic hematoma detected in the second trimester. J Obstet Gynaecol Can. 2011;33(5):475-9.

10 Winkelhorst D, Oepkes D. Foetal and neonatal alloimmune thrombocytopenia. Best Pract Res Clin Obstet Gynaecol. 2019;58:15-27. 